\title{
Estructuras de codificación del conocimiento en los postgrados de las instituciones universitarias
}

\section{Codification structures of knowledge in postgraduates of university institutions}

\begin{tabular}{|r|r|}
\hline Carlos Negrelli & Nelsy Vera \\
\cline { 3 - 3 } & revistas@cetbolivia.org \\
ORCID: 0000-0003-4510-4156 \\
Universidad del Zulia \\
Maracaibo - Venezuela
\end{tabular}$\quad \begin{array}{r}\text { ORCID: 0000-0001-5265-4290 } \\
\text { Centro de Investigación y Desarrollo } \\
\text { Guayaquil - Ecuador }\end{array}$

Artículo recibido en septiembre 2020 / Arbitrado en octubre 2020 / Aceptado en diciembre 2020 / Publicado en septiembre 2021

RESUMEN

Palabras clave:

ABSTRACT

Keywords:
Se identificaron las estructuras de codificación del conocimiento aplicados en los postgrados de las instituciones universitarias públicas de la Costa Oriental del Lago, bajo una metodología descriptiva, con diseño de campo, no experimental y transversal. La información se recolecto a través de un cuestionario contentivo de 9 ítems, con escala tipo Likert, siendo los informantes 37 profesores investigadores que dictan clases en los postgrados, y que a la vez estén activos como investigadores adscritos a algún centro de investigación. El cuestionario fue validado por expertos y la confiabilidad calculada por la formula Alfa de Cronbach cuyo resultado fue 0,92. El análisis de datos se realizó con base a la frecuencia relativa y la media aritmética. Se concluye que existe alta aplicación de la planificación y estructuración del conocimiento, captura del conocimiento y codificación del conocimiento en sistemas que permiten el desarrollo de la gestión del conocimiento.

Captura del conocimiento, codificación del conocimiento en sistemas, estructuras de codificación del conocimiento, gestión del conocimiento, planificación y estructuración del conocimiento

knowledge coding structures applied in the postgraduate courses of the public university institutions of the Eastern Coast of the Lake were identified, under a descriptive methodology, with a field design, not experimental and transversal. The information was collected through a questionnaire containing 9 items, with a Likert-type scale, with the informants being 37 research professors who teach classes in postgraduate studies, and who are at the same time active as researchers attached to a research center. The questionnaire was validated by experts and the reliability calculated by Cronbach's Alpha formula, whose result was 0.92. Data analysis was performed based on relative frequency and arithmetic mean. It is concluded that there is a high application of knowledge planning and structuring, knowledge capture and knowledge coding in systems that allow the development of knowledge management.

Knowledge capture, knowledge coding in systems, knowledge coding structures, knowledge management, knowledge planning and structuring 


\section{INTRODUCCIÓN}

La idea de una enseñanza de calidad que satisfaga a los usuarios del sistema universitario es el estandarte del nuevo modelo de universidad del siglo XXI. Por tal razón estas organizaciones deben implementar nuevas metodologías que se adapten a las distintas situaciones, intereses, ritmos de aprendizaje y circunstancias particulares.

Desde esta perspectiva, la necesidad del paso de la sociedad de la información a la del conocimiento se materializa a través de la gestión del conocimiento, convirtiéndose en un punto de presión y referencia para el desarrollo de los sistemas educativos modernos; una parte como un soporte para aprender y aplicar conocimientos, como también, para crear nuevos y por la otra, para mantenerse en coherencia o sintonía con lo que determinan las características de la educación del siglo XXI.

Así lo manifiesta la Conferencia Mundial sobre la Educación Superior (2009, p. 3) realizada en la Sede de la UNESCO, en Paris:

Los establecimientos de enseñanza superior del mundo entero tienen la responsabilidad social de contribuir a reducir la brecha en materia de desarrollo mediante el aumento de la transferencia de conocimientos a través de las fronteras, en particular hacia los países en desarrollo, y de tratar de encontrar soluciones comunes para fomentar la circulación de competencias.

En este orden de ideas, los objetivos que han dado base a la gestión del conocimiento, aplicables en las instituciones universitarias según Pavez (2003), son formular e implantar estrategias de alcance organizacional para el desarrollo, adquisición y aplicación del conocimiento; promover la mejora continua de los procesos de negocio, enfatizando la generación así como la utilización del conocimiento; monitorear y evaluar los logros obtenidos mediante la aplicación del conocimiento; reducir los tiempos de ciclos en el desarrollo de nuevos productos, mejoras de los ya existentes, la reducción del desarrollo de soluciones a los problemas además de reducir los costos asociados a la repetición de errores.

En el mismo tenor, Lugo (2008), así como también Reyes (2008), establecen que la gestión del conocimiento es una política encargada de preservar, identificar, analizar y diseminar el saber en las organizaciones, con la premisa de brindar la información adecuada a la persona correcta, en el momento oportuno, para propiciar su creatividad y excelencia.

De allí que, en las organizaciones, ha surgido un nuevo modelo gerencial conocido como gestión del conocimiento. Este busca una gestión efectiva entre los componentes intangibles del capital humano y las actividades productivas de dicha organización, para lograr mantenerse con ventajas competitivas, impactar en la sociedad y no desaparecer en el tiempo, convirtiéndose en empresas líderes en producción, intercambio de información y la incorporación de innovaciones como un activo estratégico en las organizaciones.

En ese sentido, cabe señalar lo citado por Hincapié (2009), al considerar la importancia de aquellas organizaciones que logran adquirir trascendencia porque producen, apropian y difunden conocimiento. En consecuencia, para el citado autor, las instituciones universitarias, como organización medular de las sociedades en vanguardia, en su principio epistémico deben manejar el conocimiento avanzado, por medio 
de sus tres actividades principales: la investigación, la docencia y la extensión.

Las instituciones universitarias venezolanas no escapan a esta realidad, y es a partir del año 2002, después de la V Reunión Nacional de Currículo para la Educación Universitaria Venezolana cuando se realizó un análisis de los escenarios para la Universidad del siglo XXI. En él se reflejó la necesidad de las instituciones universitarias de realizar esfuerzos mancomunados para llevar a cabo transformaciones, introduciendo adelantos e innovaciones que respondan de manera eficaz a los progresos y cambios determinantes de esta época, asumiendo este reto como estrategia para su consolidación en los mercados nacionales e internacionales por medio de un modelo de gestión de conocimiento a través de sus estructuras y estrategias permitiéndoles adecuar sus capacidades en el entorno donde se desenvuelvan.

En el caso específico de las instituciones universitarias públicas de la Costa Oriental del Lago, éstas han evolucionado en los últimos años hacia modelos organizacionales cuya estructura está orientada a fomentar la generación de conocimiento, donde se ha hecho imprescindible la participación de los diferentes postgrados que en la actualidad se han venido desarrollando. Al respecto, los estudios de postgrado representan para el profesional de hoy día por una parte una oportunidad de inserción en el mercado laboral y por otra el crecimiento productivo promotor de una mayor remuneración. Por otra parte, contribuye a incrementar el potencial del capital intelectual propio de una organización suscitando un desarrollo sustentado en el conocimiento de sus trabajadores.

Desde esta perspectiva, los postgrados constituyen un agente de la gestión del conocimiento, con vistas a su utilización para el desarrollo, donde es importante el establecimiento por parte de las instituciones universitarias de estrategias, que les permitan dar respuestas a las demandas del entorno, donde los programas de postgrado posean un rol protagónico relevante para enfrentar los desafíos de los nuevos paradigmas organizacionales.

Sobre las bases de las ideas expuestas, los investigadores a través de la técnica de la observación, además de la revisión documental de algunas estadísticas lograron constatar que los postgrados de las instituciones universitarias públicas de la Costa Oriental del Lago poseen un alto número de profesores investigadores, moderada inversión destinada a la adquisición de las tecnologías de información y comunicación, poca existencia de grupos multidisciplinarios de investigadores y una reciente estrategia de adiestramiento y capacitación hacia la comunidad universitaria en relación al abordaje de generación de conocimiento incluyendo a los estudiantes de cuarto nivel para permitir encarar la demanda real de conocimiento en su área de influencia.

Partiendo de estas consideraciones $y$ entendiendo el importante valor agregado que genera para las instituciones universitarias el conocimiento, se hace necesario identificar las estructuras de codificación, como dimensión de la variable: gestión del conocimiento. 


\section{Estructuras de codificación conocimiento}

Según Pavez (2003), la gestión del conocimiento debe ser entendida como la instancia de gestión mediante la cual se obtiene, despliega o utiliza una variedad de recursos básicos para apoyar el desarrollo del conocimiento dentro de la organización. A su vez, Udaondo (2005) afirma que la gestión del conocimiento se puede definir como el esfuerzo de una organización por conseguir, organizar, distribuir y compartir los conocimientos entre todos los empleados.

Ambos autores coinciden al considerar que la forma de estructurar las iniciativas de gestión de conocimiento generará una ventaja a la hora de considerar al conocimiento dentro de la estrategia de la administración; aspectos que permiten delinear la investigación.

De manera que, el conocimiento adquirido de una u otra forma, se pueden representar por estructuras que lo esquematicen para que sea de fácil entendimiento para los miembros de la institución. Según Nonaka y Takeuchi (1999), las estructuras de codificación están presentes en la segunda forma de conversión de conocimiento: la exteriorización, que transforma conocimiento tácito a explícito a través de la creación de conceptos nuevos. Esto prácticamente está muy relacionado con lo que expresan Davenport y Prusak (2001), cuando señalan que la codificación es convertir el conocimiento tácito en un código que no necesariamente tiene que ser informático.

Uno de los principales propósitos de este proceso es poner el conocimiento institucional al alcance de quienes lo necesitan. Para este tema de estudio en particular, un ejemplo de codificación son las leyes, reglamentos y normas internas para los diferentes procesos académicos y administrativos de las instituciones universitarias, pero que a través de ellas no se pude medir cuanto conocimiento tácito quedó sin codificar.

Como el conocimiento se genera en una constante dinámica, es muy ambicioso e inútil tratar de codificarlo en toda su amplitud, es por ello que se debe estudiar con detalle a cuáles objetivos organizacionales y estratégicos servirá el conocimiento codificado (importancia del conocimiento), así como el tipo de conocimiento explícito, tácito o intermedio (que orienta hacia qué hacer con el conocimiento). Es decir, que el conocimiento tácito se codifica de diferentes maneras al conocimiento explícito.

Esta parte de la investigación solo abordará las formas de representación del conocimiento tácito, por la importancia fundamental que tiene en la generación de nuevo conocimiento $y$ porque tal vez nunca se haya codificado bajo una estructura formal, cuestión que no ha sucedido con el conocimiento explícito debido a su propia naturaleza (al ser explícito sugiere una codificación previa); es por ello que sería interesante solamente evaluarlo y luego ponerlo a disponibilidad de toda los postgrados de las instituciones universitarias públicas de la Costa Oriental del Lago.

En tal sentido, a objeto de esta investigación, la codificación en las organizaciones adapta el conocimiento a formato accesible y aplicable. Los administradores y usuarios del conocimiento pueden catalogarlo, describirlo, esquematizarlo, moldearlo e incorpóralos en normas y recetas y será analizado a través de la planificación y estructuración del conocimiento, captura del conocimiento y codificación del conocimiento en sistemas. 


\section{Planificación y estructuración del conocimiento}

La planificación y estructuración del conocimiento consiste en diseñar unas herramientas con el fin de codificar el conocimiento tácito, tal vez algún conocimiento confuso pero que se requiera organizar porque es muy importante para cierto proceso administrativo de la institución. Para Davenport y Prusak (2001), la planificación del conocimiento tácito, entonces se limitará a encontrar las personas que tengan el conocimiento, enviar a quién está buscándolo y estimular la interacción valiéndose para ello de unas herramientas estructuradas para tal fin.

En este contexto, Valhondo (2003), indica que una gran parte del conocimiento humano es tácito, siendo especialmente cierto en las competencias operativas, así como en el saber práctico adquirido por la experiencia; el de este tipo se orienta a la acción, siendo personal, lo cual hace difícil su formalización y comunicación.

Por otro lado, Vega (2004), argumenta que el conocimiento constituye la información que posee valor para la organización, siendo el tácito aquel de difícil articulación de manera que sea completamente manejable; adicionalmente, indica que se trata del adquirido mediante la experiencia en los procesos organizacionales.

En similar referencia, Paniagua (2007), lo define como aquel de dificultosa expresión, en razón de lo cual no se encuentra codificado; encontrándose dentro de esta categoría las experiencias de trabajo, emocionales, vivenciales, las habilidades, las creencias, las destrezas, entre otras de similar naturaleza. Mientras que el conocimiento En referencia a este conocimiento explicito es aquel codificado, siendo factible transmitirlo a través de algún sistema de lenguaje formal; ubicando en esta categoría los documentos, reportes, memos, mensajes, presentaciones, diseños, especificaciones, simulaciones, entre otros destacados.

En opinión de Valhondo (2003), este tipo de conocimiento se puede generar por medio de deducciones lógicas y se adquiere a través del estudio formal; en contraposición a ello, el tácito solamente se puede adquirir a través de la experiencia práctica en un contexto relevante, vale decir, aprender haciendo.

Por su parte, Davenport y Prusak (2001) proponen las siguientes alternativas para la planificación y estructuración del conocimiento:

Mapas: Son estructuras que agilizan la búsqueda de quién tiene verdaderamente el conocimiento. Pueden ser de varias formas: mapa real, páginas amarillas, una base de datos (por ejemplo, como la base de datos de los intercambios verbales o escritos de las redes o groupware o la base de datos de recursos humanos), organigramas, paquetes y sistemas de recursos humanos, guías telefónicas, entre otras; que pueden referirse también a personas o documentos especializados.

Tal vez la institución cuente con el organigrama como principal mapa de conocimiento, los cuales tienen la particularidad de ser estrictamente verticales y destacan con más detalle la parte gerencial que la funcional u operativa, pero el conocimiento clave puede estar en cualquier nivel de esta estructura; los mapas de conocimiento no subestiman ningún nivel para el conocimiento y reflejan los conocimientos adquiridos.

Tecnología de planificación del conocimiento: Se refiere al diseño electrónico de estos instrumentos $y$ al uso de las computadoras para mejorar los formatos de 
presentación de los mapas de conocimiento añadiéndole imágenes de la persona, breves videos o una personalización más amplia de los datos contenidos con otro tipo de información.

Así mismo, los programas de
comunicaciones como groupware y
navegadores web para los sistemas de páginas amarillas electrónicas. Otro mapa de conocimiento pudiera estar inmerso en los paquetes de recursos humanos, que generalmente se usan para nómina de empleados pueden además usarlo para relacionar remuneraciones con el conocimiento, ofreciendo categorías de conocimiento simples y genéricas. En cambio, con los sistemas de recursos humanos conectados o no en interfaz con sistemas más amplios; son usados principalmente para la contratación de personal externo; puede aumentar la categoría de conocimiento, aunque sigue siendo genérica y limitada.

Políticas de planificación del conocimiento: el reconocimiento y la recompensa son políticas de planificación del conocimiento y el mapa además de ser un medio para ubicar el conocimiento, reflejará la importancia que representa para la organización la fuerza laboral. Con esta dinámica del proceso, se pueden generar disputas por ocupar los niveles de mayor jerarquía, que también serán una señal de la importancia del conocimiento para la organización.

Estructuración del conocimiento: La estructuración del conocimiento se estudiará a partir de la identificación de obstáculos que evitan el flujo y la utilización del conocimiento, y no desde el punto de vista del valor del aporte del conocimiento como tal, además de todas las dificultades que implica codificar el conocimiento tácito o implícito. Entonces, así será importante identificar las variables del modelo de codificación que pueden afectarse debido a las operaciones administrativas de la institución, tales como fallas de infraestructura, impedimentos sociales, políticos, cognitivos y comunicativos; y defectos en la voluntad administrativa.

A juicio de los investigadores, para algunas de estas maneras usadas para la codificación del conocimiento tácito, es indispensable la disposición del personal para compartir el conocimiento. Para algunos tipos de mapas es importante conocer el nivel académico del personal, sobre este aspecto estos autores destacan que el título o cargo es una señal formal de quién debería tener conocimientos valiosos, pero a veces no es suficiente, ya que es posible que la persona reseñada en el organigrama este desactualizada en la información requerida o bien no esté dispuesta a compartirla. Es por ello entonces, que el organigrama de la institución no es un instrumento eficaz para determinar donde reside el conocimiento clave.

\section{Captura del conocimiento}

En este contexto, el término capturar se refiere a disponer del conocimiento tácito cuando se necesite, se ubique o no en la institución la persona que lo tiene. Consiste en apoderarse del conocimiento a través del registro de historias contadas por los expertos y de la incorporación del conocimiento en leyes, normas y reglamentos; para lo que se usan nuevamente las tecnologías de información y comunicación. Son formas fáciles y rápidas de codificar el conocimiento tácito.

Para Valhondo (2003) los datos pueden convertirse en información cuando se le se añade sentido mediante los métodos 
contextualizados, es decir cuando tienen un propósito; categorizados como componentes claves; calculados a través de procesos estadísticos; corregidos, cuando se eliminan datos erróneos; y condensados o resumidos.

La información por su parte tiene sentido, proporciona juicio e interpretación el cual facilita el proceso de la toma de decisiones, pues está organizada con algún propósito, en efecto, el aprendizaje en las organizacional y la eficacia en su gestión dependen del óptimo manejo de la información.

En otro orden de idas, Rodríguez (2006) plantea que los procesos de almacenamiento, acceso y transferencia de conocimiento, son partes del proceso general que encierra la gestión del conocimiento, el cual poco se distingue el conocimiento de la información y los datos porque actúan integralmente entre las personas que lo crean y lo utilizan. En la gestión del conocimiento estos elementos se centran en el desarrollo de metodologías, estrategias y técnicas para almacenar el conocimiento disponible en la organización, en depósitos de fácil acceso para propiciar su posterior transferencia entre los miembros de la organización.

Sin embargo, Quiroga (2002) afirma que la captura de información: comprende las actividades relacionadas con la obtención de la información adecuada, en el tiempo y lugar adecuado, para tomar la decisión adecuada. De acuerdo a lo que señala Senge (2002), la gestión del conocimiento, como proceso de identificación, captura, organización y diseminación de los datos claves y la información necesaria para ayudar a la organización a responder a las necesidades de los clientes, busca la perpetuación y la materialización del potencial de las organizaciones.
Por su parte, Davenport y Prusak (2001) proponen las siguientes alternativas para la captura del conocimiento:

Valor de la narrativa: Las narrativas son historias contadas a través de una persona elocuente que use la retórica para contar verdades prácticas, las mismas pueden codificarse tratando de aprovechar el valor sustancial del conocimiento. La experiencia del orador debe ser tanto para transmitir lo que realmente sucede y no lo que debería suceder con el conocimiento, como para comunicarse de tal manera que los demás empleados los puedan entender.

Cabe destacar, que este tipo de transmisión del conocimiento (la narrativa, el discurso y la oratoria) es característico de las instituciones de educación tales como las universidades. Algunas organizaciones distribuyen y proyectan estos discursos entre sus empleados a través de la vía electrónica o videos. Algunas utilizan las redes para emitir información útil en forma de historia que necesita que conozcan sus empleados. Esta manera de capturar el conocimiento por medio de la narrativa permitirá la disponibilidad del mismo las veces que sea necesario

Conocimiento incorporado: En la dinámica laboral, los empleados expertos incorporan conocimiento a los procesos, productos o servicios de una organización, procesos que no se detienen, aunque ya el empleado no pertenezca a la institución. Sin embargo, es muy probable que, si esto sucede, el proceso no funcione de la misma manera y afecte el producto o servicio esperado, porque este personal no puede dejar en ese conocimiento explícito la capacidad y destreza con la que ejecutaba el proceso.

Por lo antes mencionado se observa la relevancia que tiene dentro de los postgrados 
de las instituciones universitarias públicas de la Costa Oriental del Lago, la gestión del conocimiento y con ella la captura de la información que permite conocer tanto dentro y fuera de la institución los elementos importantes para aplicarlos y elevar su nivel de competitividad.

\section{Codificación del conocimiento en sistemas}

La codificación del conocimiento se define como el proceso de conversión del conocimiento tácito codificable en mensajes, patentes, bases de datos, manuales de procedimientos, que pueden ser procesados a partir de ese momento como información (Balconi, 2002).

Esta forma de representar el conocimiento es factible, ya que en las instituciones universitarias se ejecutan procesos rutinarios que los expertos le han agregado valor, que se pueden diseñar como sistemas expertos o bien adaptar uno a alguno de los procesos académico-administrativo de unas instituciones de estas características.

En efecto, diseñar un sistema como estos implica gran consumo de tiempo e intensas horas de trabajo, sobre todo en la extracción de conocimiento a expertos humanos para posteriormente codificarlo en los sistemas expertos correspondientes. Además, requiere de personal de tecnología capacitado en el área de inteligencia artificial a través de programas de adiestramiento, cursos, servicios de consultoría, y la práctica diaria.

Según Davenport y Prusak (2001), los sistemas expertos son una forma de representar y organizar el conocimiento por medio de programas de computación que incluyen los conocimientos de expertos humanos en un área de especialización. Son de gran utilidad para convertir conocimiento tácito a explícito y de explícito a explícito.

Cuando el proceso o producto por muy complejo que sea, esté basado en una serie de reglas, normas y procedimientos estáticos y cerrados, tiene posibilidades de codificarse al ser transformado o imitado. Ya existen sistemas expertos, en algunas oportunidades pudiera adaptarse uno a las necesidades de la organización, en este caso a los postgrados de las instituciones universitarias públicas de la Costa Oriental del Lago.

A los fines investigativos, la codificación del conocimiento permite poner el conocimiento institucional en un código con la finalidad de organizarlo en forma de bases de datos e informes escritos, informes video-conferencia y de sonido para hacerlo explícito, portable y fácil de entender: ponerlo a la disposición de quién lo necesite.

La codificación asociada a la transferencia del conocimiento, involucra a la transmisión y absorción (uso) del conocimiento. El enfoque presentado se orienta a la formación de redes de relaciones, vinculado a la socialización en términos de Nonaka y Takeuchi (1999), y su desarrollo y comercialización íntimamente ligada a consideraciones socio-políticas de las redes inter-organizacionales.

\section{MÉTODO}

La metodología empleada fue de tipo descriptiva, con un diseño de campo, no experimental y transversal. La información se recolecto a través de la aplicación de un cuestionario contentivo de 9 ítems, con escala tipo Likert, siendo los informantes 37 profesores investigadores que dictan clases en los postgrados de las instituciones universitarias de 
la COL, y que a la vez estén activos como investigadores adscritos a algún centro de investigación. El cuestionario fue validado por cinco expertos y la confiabilidad calculada por la formula Alfa de Cronbach cuyo resultado fue 0,92 indicando muy alta confiabilidad.

El análisis de datos se realizó con base a la frecuencia relativa y la media aritmética. Para el análisis de las frecuencias se asumió que la suma de las frecuencias relativas de las alternativas (Siempre (S) + Casi siempre (CS)) para los indicadores cuando supera el $75 \%$ se considera como tendencia positiva la gestión del conocimiento, tal como se aprecia en el cuadro 1.

Cuadro 1. Categoría de análisis para la frecuencia relativa

\begin{tabular}{cc}
\hline Alternativa & Tendencia \\
\hline TA $+\mathrm{DA} \geq 75 \%$ & La tendencia se considera positiva \\
$45 \% \leq \mathrm{TA}+\mathrm{DA}<75 \%$ & La tendencia se considera neutral \\
$\mathrm{TA}+\mathrm{DA}<45 \%$ & La tendencia se considera negativa \\
\hline
\end{tabular}

Fuente: Elaboración propia (2019)

Mientras que, para la media aritmética, se estableció un baremo, basado en escala de alternativas de respuestas utilizada en el instrumento (Likert), para su interpretación fue diseñado por los investigadores un cuadro de rango, intervalo y categoría, de manera de lograr la interpretación del análisis de los resultados de las dimensiones que se presentan a continuación. En el cuadro 2 se muestra el baremo para el análisis de la media aritmética.

Cuadro 2. Categoría de análisis para la interpretación del promedio

\begin{tabular}{ccc}
\hline Dimensión & Rango para la media & $\begin{array}{c}\text { Nivel de respuesta para la dimensión } \\
\text { y sus indicadores }\end{array}$ \\
\hline Estructuras de & $4.20 \leq \bar{\chi} \leq 5.00$ & Muy alta aplicación \\
codificación del & $3.40 \leq \bar{\chi}<4.20$ & Alta aplicación \\
conocimiento & $2.60 \leq \bar{\chi}<3.40$ & Moderada aplicación \\
& $1.80 \leq \bar{\chi}<2.60$ & Baja aplicación \\
& $1.00 \leq \bar{\chi}<1.80$ & Muy baja aplicación \\
\hline
\end{tabular}

Fuente: Elaboración propia (2019)

\section{RESULTADOS}

La tabla 1 muestra los resultados en función del indicador: planificación y estructuración del conocimiento, la cual arroja agrupación de respuestas para los ítems 1, 2 y 3 en las opciones totalmente de acuerdo y de acuerdo, cuyos valores ascienden a $60 \%, 73 \%$ y
70\% respectivamente, marcando tendencia neutral para todos los ítems. Se observa un promedio de respuestas en la categoría totalmente de acuerdo y de acuerdo del $68 \%$, indicando tendencia neutral en las respuestas obtenidas. 
Tabla 1. Indicador: Planificación y estructuración del conocimiento

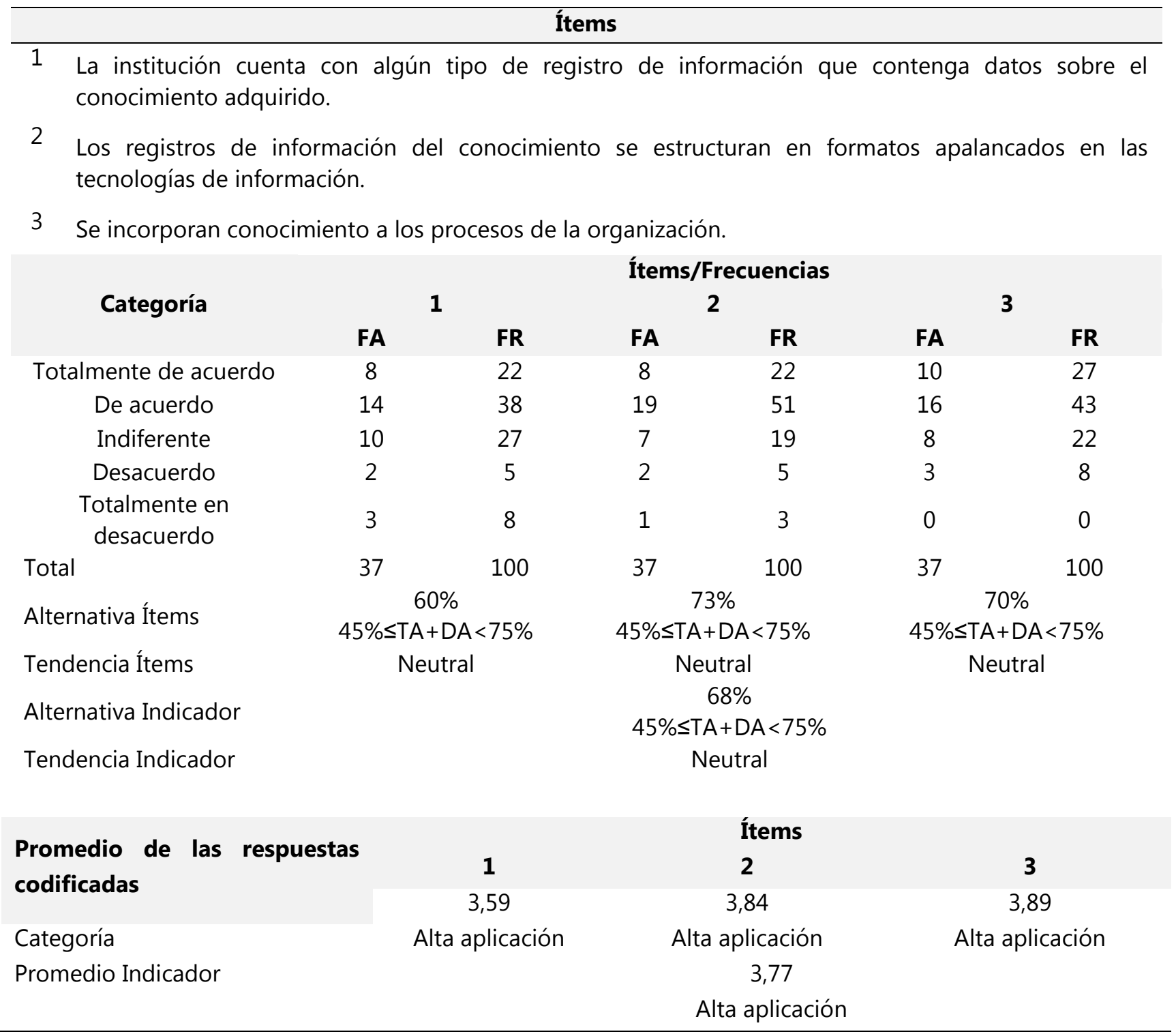

Fuente: Elaboración propia (2019)

Adicional a ello, se evidencia un promedio de 3,77 ubicándolo en la categoría de alta aplicación. Al detalle se obtuvo que todos los ítems se posicionaron en la categoría de alta aplicación (3,59; 3,84 y 3,89; respectivamente) afirmando que los siguientes aspectos presentan alta aplicación: la institución cuenta con algún tipo de registro de información que contenga datos sobre el conocimiento adquirido, los registros de información del conocimiento se estructuran en formatos apalancados en las tecnologías de información y se incorporan conocimiento a los procesos de la organización.

Estos resultados permiten considerar que en los postgrados de las instituciones universitarias púbicas objeto de estudio se diseñan herramientas con el fin de codificar el conocimiento, tal como lo afirma Davenport y Prusak, (2001), de tal forma que sea asequible y de fácil comprensión para quién lo necesita. 
Con base a los resultados obtenidos para los investigadores en los postgrados de las instituciones analizadas la codificación del conocimiento debería ocurrir en la formulación escrita y participativa de proyectos, en donde se reflejen dentro de la estrategia y actividades los aprendizajes que se han tenido en experiencias previas, en tal sentido, la tarea de los administradores o gestores del conocimiento es bajar el conocimiento a un lenguaje o código que sea familiar y comprensible para el resto de los interesados.

Respecto al indicador captura del conocimiento, los resultados se muestran en la tabla 2, evidenciándose en cada uno de los ítems aplicados 4; 5 y 6 , cuyos valores agrupados en las opciones totalmente de acuerdo y de acuerdo alcanzan valores del $46 \%$; $59 \%$ y $68 \%$, marcando tendencia neutral, por tanto, en promedio el indicador se ubicó también en una tendencia neutral con un valor de $58 \%$.

Al mismo tiempo, se observa en la tabla señalada que los ítems se posicionaron en la categoría de alta aplicación $(3,72)$, afirmando los encuestados que se utilizan las tic's para registrar las historias de los expertos, en lo que se refiere a su experiencia en los procesos de la institución $(3,46)$; la institución utiliza las redes para emitir información útil que necesita que conozcan sus investigadores $(3,92)$ y se distribuye el conocimiento generado a través de la vía electrónica $(3,78)$.

El resultado obtenido es indicador de que, en los postgrados de las instituciones universitarias públicas objeto de estudio la captura del conocimiento comprende las actividades relacionadas con la obtención de la información adecuada, en el tiempo y lugar adecuado, para tomar la decisión adecuada, validando así lo expuesto por Quiroga (2002). A criterio de los investigadores, la captura y almacenamiento determinan en buena parte la eficacia de un sistema de gestión del conocimiento.

Tabla 2. Indicador: Captura del conocimiento

\section{Ítems}

4 Se utilizan las tic's para registrar las historias de los expertos, en lo que se refiere a su experiencia en los procesos de la institución.

5 La institución utiliza las redes para emitir información útil que necesita que conozcan sus investigadores.

6 Se distribuye el conocimiento generado a través de la vía electrónica. 


\begin{tabular}{|c|c|c|c|c|c|c|}
\hline \multirow{3}{*}{ Categoría } & \multicolumn{6}{|c|}{ Ítems/Frecuencias } \\
\hline & \multicolumn{2}{|c|}{4} & \multicolumn{2}{|c|}{5} & \multicolumn{2}{|c|}{6} \\
\hline & FA & FR & FA & $\mathbf{F R}$ & FA & FR \\
\hline Totalmente de acuerdo & 8 & 22 & 13 & 35 & 8 & 22 \\
\hline De acuerdo & 9 & 24 & 9 & 24 & 17 & 46 \\
\hline Indiferente & 13 & 35 & 14 & 38 & 9 & 24 \\
\hline Desacuerdo & 6 & 16 & 1 & 3 & 2 & 5 \\
\hline $\begin{array}{c}\text { Totalmente en } \\
\text { desacuerdo }\end{array}$ & 1 & 3 & 0 & 0 & 1 & 3 \\
\hline Total & 37 & 100 & 37 & 100 & 37 & 100 \\
\hline \multirow[t]{2}{*}{ Alternativa Ítems } & \multicolumn{2}{|c|}{$46 \%$} & \multicolumn{2}{|c|}{$59 \%$} & \multicolumn{2}{|c|}{$68 \%$} \\
\hline & \multicolumn{2}{|c|}{$45 \% \leq T A+D A<75 \%$} & \multicolumn{2}{|c|}{$45 \% \leq T A+D A<75 \%$} & \multicolumn{2}{|c|}{$45 \% \leq \mathrm{TA}+\mathrm{DA}<75 \%$} \\
\hline \multirow{3}{*}{$\begin{array}{l}\text { Tendencia Ítems } \\
\text { Alternativa Indicador }\end{array}$} & \multicolumn{2}{|c|}{ Neutral } & \multicolumn{2}{|c|}{ Neutral } & \multicolumn{2}{|c|}{ Neutral } \\
\hline & & & \multicolumn{2}{|c|}{$58 \%$} & & \\
\hline & \multicolumn{6}{|c|}{$45 \% \leq T A+D A<75 \%$} \\
\hline Tendencia Indicador & \multicolumn{6}{|c|}{ Neutral } \\
\hline \multirow{3}{*}{$\begin{array}{c}\text { Promedio de las respuestas } \\
\text { codificadas }\end{array}$} & \multicolumn{6}{|c|}{ Ítems } \\
\hline & \multicolumn{2}{|c|}{4} & \multicolumn{2}{|c|}{5} & \multicolumn{2}{|c|}{6} \\
\hline & \multicolumn{2}{|c|}{3,46} & \multicolumn{2}{|c|}{3,92} & \multicolumn{2}{|c|}{3,78} \\
\hline Categoría & & cación & & ación & & \\
\hline \multirow[t]{2}{*}{ Promedio Indicador } & \multicolumn{6}{|c|}{3,72} \\
\hline & \multicolumn{6}{|c|}{ Alta aplicación } \\
\hline
\end{tabular}

Fuente: Elaboración propia (2019)

En cuanto al análisis de la codificación del conocimiento en sistemas representado en la tabla 3, se observa en los resultados de los ítems 22,23 y 24 frecuencias de $60 \%$, y $51 \% \%$ respectivamente, concentradas en las opciones totalmente de acuerdo y de acuerdo, con tendencia neutral. En términos generales arrojo un promedio para el indicador de $54 \%$, con tendencia neutral.

Adicionalmente, la tabla muestra un promedio de 3,65 lo cual según el baremo establecido ubica al indicador en la categoría de alta aplicación. Esto se refleja en sus medias de $3,68,3,65$ y 3,62 respectivamente, indicando según opinión de los encuestados alta aplicación de que los procesos académicos de la institución puedan diseñarse a través de un sistema experto y a un sistema experto ya diseñado. De igual manera, que se asigna un código al conocimiento institucional con la finalidad de organizarlo en forma de bases de datos. 
Tabla 3. Indicador: Codificación del conocimiento en sistemas

\begin{tabular}{|c|c|c|c|c|c|c|}
\hline \multicolumn{7}{|c|}{ Ítems } \\
\hline \multirow{3}{*}{\multicolumn{7}{|c|}{$\begin{array}{l}\text { Los procesos académicos de la institución puedan diseñarse a través de un sistema experto. } \\
\text { Los procesos académicos de la institución, pudiera adaptarse a un sistema experto ya diseñado. } \\
\text { Se asigna un código al conocimiento institucional con la finalidad de organizarlo en forma de bases de } \\
\text { datos. }\end{array}$}} \\
\hline & & & & & & \\
\hline & & & & & & \\
\hline \multirow{3}{*}{ Categoría } & \multicolumn{6}{|c|}{ Ítems/Frecuencias } \\
\hline & \multicolumn{2}{|c|}{7} & \multicolumn{2}{|c|}{8} & \multicolumn{2}{|c|}{9} \\
\hline & FA & FR & FA & FR & FA & FR \\
\hline Totalmente de acuerdo & 5 & 14 & 7 & 19 & 9 & 24 \\
\hline De acuerdo & 17 & 46 & 12 & 32 & 10 & 27 \\
\hline Indiferente & 13 & 35 & 16 & 43 & 14 & 38 \\
\hline Desacuerdo & 2 & 5 & 2 & 5 & 3 & 8 \\
\hline Totalmente en desacuerdo & 0 & 0 & 0 & 0 & 1 & 3 \\
\hline Total & 37 & 100 & 37 & 100 & 37 & 100 \\
\hline \multirow{2}{*}{ Alternativa Ítems } & \multicolumn{2}{|c|}{$60 \%$} & \multicolumn{2}{|c|}{$51 \%$} & \multicolumn{2}{|c|}{$51 \%$} \\
\hline & \multicolumn{2}{|c|}{$45 \% \leq T A+D A<75 \%$} & \multicolumn{2}{|c|}{$45 \% \leq T A+D A<75 \%$} & \multicolumn{2}{|c|}{$45 \% \leq T A+D A<75 \%$} \\
\hline Tendencia Ítems & \multicolumn{2}{|c|}{ Neutral } & \multicolumn{2}{|c|}{ Neutral } & \multicolumn{2}{|c|}{ Neutral } \\
\hline \multirow{2}{*}{ Alternativa Indicador } & \multicolumn{6}{|c|}{$54 \%$} \\
\hline & \multicolumn{6}{|c|}{$45 \% \leq T A+D A<75 \%$} \\
\hline Tendencia Indicador & \multicolumn{6}{|c|}{ Neutral } \\
\hline \multirow{3}{*}{$\begin{array}{l}\text { Promedio de las respuestas } \\
\text { codificadas }\end{array}$} & \multicolumn{6}{|c|}{ Ítems } \\
\hline & \multicolumn{2}{|c|}{7} & & & \multicolumn{2}{|c|}{9} \\
\hline & \multicolumn{2}{|c|}{3,68} & \multicolumn{2}{|c|}{3,65} & \multicolumn{2}{|c|}{3,62} \\
\hline Categoría & \multicolumn{2}{|c|}{ Alta aplicación } & \multicolumn{2}{|c|}{ Alta aplicación } & Alta & \\
\hline Promedio Indicador & & & & & & \\
\hline & & & Alt & ción & & \\
\hline
\end{tabular}

Fuente: Elaboración propia (2019)

Según estos resultados, en los postgrados de las instituciones analizadas, se aplican los sistemas de expertos como una forma de representar y organizar el conocimiento por medio de programas de computación que incluyen los conocimientos de expertos humanos en un área de especialización, aspectos que se evidencian en la teoría expuesta por Davenport y Prusak, (2001). En este sentido, a criterio de los investigadores el conocimiento se estructura en una unidad simple o compuesta que se clasifica según géneros y atributos; y su valor es función del grado de utilización y rol desempeñado desde perspectivas del actor individual y la organización. 
Ya analizados cada uno de los indicadores considerados en la dimensión denominada estructuras de codificación del conocimiento, se pasa a verificar los resultados arrojados para esta dimensión. En la tabla 4 se observa tendencia neutral con una media que permite ubicar a esta dimensión en la categoría de alta aplicación, así entonces se observó que cada uno de los componentes referidos a: planificación y estructuración del conocimiento, captura del conocimiento y codificación del conocimiento en sistemas permiten el desarrollo de la gestión del conocimiento.

Tabla 4. Dimensión: Estructuras de codificación del conocimiento

\begin{tabular}{ccccc}
\hline \multicolumn{1}{c}{ Indicador } & Alternativa & Categoría & Media & Categoría \\
\hline $\begin{array}{l}\text { Planificación y estructuración } \\
\text { del conocimiento } \\
\text { Captura del conocimiento }\end{array}$ & $\begin{array}{c}68 \% \\
45 \% \leq \mathrm{TA}+\mathrm{DA}<75 \%\end{array}$ & Neutral & 3,77 & Alta aplicación \\
$\begin{array}{c}58 \% \\
45 \% \leq \mathrm{TA}+\mathrm{DA}<75 \%\end{array}$ & Neutral & 3,72 & Alta aplicación \\
$\begin{array}{c}54 \% \\
\text { Codificación del conocimiento }\end{array}$ & $\begin{array}{c}\text { Neutral } \\
\text { en sistemas }\end{array}$ & 3,65 & Alta aplicación \\
\multicolumn{1}{c|}{$\begin{array}{c}\mathbf{6 0 \%}+\mathrm{DA}<75 \% \\
\text { Promedio }\end{array}$} & Neutral & $\mathbf{3 , 7 1}$ & Alta aplicación \\
\hline
\end{tabular}

Fuente: Elaboración propia (2019)

Según estos resultados, el conocimiento adquirido en los postgrados de las instituciones analizadas, de una u otra forma se puede representar por estructuras que lo esquematicen para que sea de fácil entendimiento entre sus miembros, siendo estos resultados congruentes con la teoría referida por Nonaka y Takeuchi (1999), en ellos se convierte el conocimiento tácito en un código que no necesariamente tiene que ser informático, aspectos manejado por Davenport y Prusak (2001).

A criterio de los investigadores, el uso de las estructuras de codificación del conocimiento ha dado lugar a un gran abanico de herramientas cuya finalidad es soportar estas estructuras $y$ facilitar los flujos de conocimiento entre los agentes que las componen. De manera que, en los postgrados de las instituciones no sólo se debe disponer de medios tecnológicos para la generación, síntesis y transmisión del conocimiento.

\section{CONCLUSIONES}

En lo que concierne al objetivo, dirigido a identificar las estructuras de codificación del conocimiento aplicados en los postgrados de las instituciones universitarias públicas de la Costa Oriental del Lago, se concluye que existe alta aplicación de la planificación y estructuración del conocimiento, captura del conocimiento y codificación del conocimiento en sistemas que permiten el desarrollo de la gestión del conocimiento, logrando con ello identificar quién tiene el conocimiento y hacerlo ubicable para interactuar con él cuando sea necesario. 
REFERENCIAS

Balconi, M. (2002). Tacitness, codification of technological knowledge, and the organization of industry. Research policy. vol 31 pp 357-379

Conferencia Mundial sobre la Educación Superior. (2009): La nueva dinámica de la educación superior y la investigación para el cambio social y el desarrollo (Sede de la UNESCO, París,5 -8 de julio de 2009). COMUNICADO.

Disponible

en http://www.extension.luz.edu.ve/

Davenport, T. y Prusak L. (2001). Conocimiento en Acción. Prentice Hall. Buenos Aires: Argentina

Hincapié, T. (2009). Gestión del Conocimiento. Ediciones Gestión 2000 S.A. Barcelona, España

Lugo, O. (2008). Procedimiento general para la implantación de un sistema de gestión del conocimiento, como fuente de ventajas competitivas sostenibles. UCLV

Nonaka, I., Takeuchi, H. (1999). La Organización Creadora del Conocimiento. The Knowledge-Creating Company. Oxford University Press. USA

Paniagua, E. (2007). La gestión tecnológica del conocimiento. Ediciones de la Universidad de Murcia
Pavez, A. (2003). Modelo de implantación de gestión del conocimiento y tecnología de información para la generación de ventajas competitivas. Valparaíso. España

Quiroga, A. (2002). Valores de Excelencia para Triunfar, Tomo I, Editorial Grad, México, D.F

Reyes, B. (2008). Comportamiento Organizacional. International Thomson Editores, S.A. México

Rodríguez, G. (2006). Modelos para la creación y gestión del conocimiento: una aproximación teórica" Disponible en http://books.google.co.ve/books

V Reunión Nacional de Curriculum para la Educación Superior (2002). Centro de Estudios en Teleinformática. Universidad Nacional Experimental del Táchira. Extraído de

http://www.unet.edu.ve/ cccunet/lineamien tos.html

Valhondo, D. (2003). Gestión del conocimiento: Del mito a la realidad. Ediciones Díaz Santos S.A

Vega, G. (2004). La era de la coevolución del conocimiento y el mundo inteligente. Ediciones Gestión del conocimiento, España 